\title{
Label-free Mie Scattering Identification of Tumor Tissue Using an Angular Photodiode Array
}

\author{
Matthew V. Bills and Jeong-Yeol Yoon \\ Department of Biomedical Engineering, The University of Arizona, Tucson, AZ 85721, USA \\ Received XX XXX 20XX, revised XX XXX 20XX, accepted XX XXX 20XX, published XX XXX 2020, current version XX XXX 20XX.
}

\begin{abstract}
Tumors differ from normal tissues in several meaningful ways including cellular size, morphology, and protein expression, which will accordingly change the refractive index and the size/morphology of cells. There are also important differences in tissue organization and unique tissue specific cell densities. Instead of time-consuming and labor-intensive histology involving the use of a benchtop microscope, a plot of Mie scattering intensities at fixed wavelength against scattering angle, which we referred to as "Mie spectrum," is suggested as an alternative to identify tumor from normal tissues. An angular photodiode array is developed to measure this Mie spectrum with three different light emitting diodes (blue, green and red) as light sources. The resulting Mie spectra show characteristic peaks for rat colonic tissues, and substantial differences can be found between tumor vs. normal tissues. Two peaks were identified at $120^{\circ}$ and $150^{\circ}$ scattering angles, potentially representing capillaries and colon cells, respectively. Contributions from crypts and goblet cells, represented by the scattering at $140^{\circ}$, were minimal. Substantial differences between tumor and normal tissues were found with $45^{\circ}-70^{\circ}$ light irradiation angles.
\end{abstract}

Index Terms—colon cancer; rat model, light scattering, tissue biopsy.

\section{INTRODUCTION}

Angle-dependent Mie scattering diagnostic and imaging modalities are rapid and can be employed noninvasively, i.e. requiring no labels. As a result, measuring Mie scatter can become a promising diagnostic method for various medical conditions such as cancer. Mie scattering occurs when the size of a scattering structure becomes comparable to or larger than the wavelength of incident light. Scattering intensities are constructively added at a certain angle while they can be destructively attenuated at the other angle, creating a highly angledependent scattering profile. Factors that alter Mie scattering are the refractive index, size, and density of scattering object, as well as the wavelength and angle of incident light. Mie scattering-based optical sensing techniques, such as the use of Mie scattering intensities at fixed angle to identify surface characteristics, are often employed during quality-control checks of manufactured surfaces and materials because optical scattering is highly informative about a surface's physical characteristics including factors such as surface roughness. Our laboratory has employed this method toward more biological matrices including identifying bacterial presence and species on a human and animal skin as well as on a wound, i.e. skin and wound infection, without using any labels [1,2]. Labels can be added to amplify such Mie scattering intensities, for example the submicronor micron-sized beads with high refractive indices such as polystyrene and $\mathrm{TiO}_{2}$. Our laboratory has employed this technique a number of times to identify samples labeled with antibody-conjugated polystyrene beads on a number of different microfluidic platforms including on polydimethylsiloxane (PDMS) and nitrocellulose substrates [3-5]. Others have labeled tumor cells with $\mathrm{TiO}_{2}$ beads and measured scattering [6] as well as for detecting and measuring fibroblast necrosis [7]. While these beads have successfully amplified the Mie scattering intensities measured at a fixed angle, it may be possible to identify the type and morphology of tissue without using such beads (or any other labels).

The method presented in this work, i.e. collecting Mie scattering intensities at multiple incident and detection angle combinations from tissue samples, does not require sample processing, beads, additional reagents, or bioreceptors, which eliminates time and labor for lengthy reagent and sample preparation. Our device consists of an array of light emitting diodes (LEDs) and photodiodes that detect Mie scattering from a tissue surface. LEDs are mounted at angles of $90^{\circ}$, $80^{\circ}, 70^{\circ}, 60^{\circ}$, and $45^{\circ}$, relative to the tissue surface and Mie scattering is detected at $10^{\circ}$ increments from $110^{\circ}$ to $170^{\circ}$ to instantly obtain a full Mie scattering spectrum (i.e. a plot of Mie scattering intensities against the scattering angle at the fixed incident wavelength), as depicted in Fig. 1.

We propose to use this method in lieu of time-consuming and laborintensive histology preparation and analysis involving the preparation of histological slides and the clinical expertise to identify specimen. Histology is one of the most definitive methods of tumor diagnosis, where a tissue sample is collected ("biopsied") from a tissue of interest and subsequent microscopic examination is made with appropriate staining. Rapid, accurate, label-free, microscope-free diagnosis of tissue samples in the clinic would be helpful in aiding clinicians to make timely and accurate diagnosis. Our device is designed to be used with biopsy samples, without the need for staining (requiring labor) and microscopy (bulky and expensive).

The specific aims of this research are 1) to identify whether the Mie spectrum can be used to identify tumors from normal tissues that are not affected by blood absorption and direct surface reflection, 2) to optimize the color and angle of light irradiation and the angle of light 
detection, and 3) to identify the tissue components that are responsible for the differences between normal vs. tumor tissues.

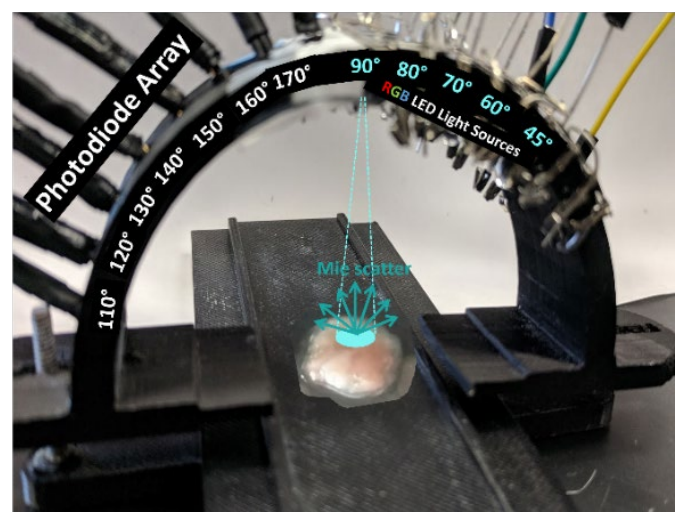

Fig. 1. Angular photodiode array positioned at $110^{\circ}-170^{\circ}$ with red, green and blue LED light sources positioned at $45^{\circ}-90^{\circ}$.

\section{MATERIALS AND METHODS}

\section{A. Sensor Design}

An angular photodiode array and tissue stage were created using SolidWorks 2013 x64 Edition (Dassault Systèmes, VélizyVillacoublay, France) and 3D-printed in ABS (acrylonitrilebutadiene-styrene) polymer using (Ultimaker 3; Ultimaker, Utretch, Netherlands). The photodiode array consists of a semi-circle (radius $=32 \mathrm{~mm}$ ) with eight insertion points for PIN photodiodes (MTD1114M3B; Marktech Optoelectronics, Latham, NY, USA) arranged in a single plane at $10^{\circ}$ increments from $110^{\circ}$ to $170^{\circ}$ relative to a light source shining perpendicular to the surface (Fig. 1). Three types of LED light sources, red $\left(624 \mathrm{~nm}, 6^{\circ}\right.$ viewing angle; 383 2SURC/S400-A6; Everlight Electronics, Shulin, Taiwan), green (505 $\mathrm{nm}, 8^{\circ}$ viewing angle; OVLGC0C6B9; TT Electronics, Woking, England), and blue (470 nm, $15^{\circ}$ viewing angle; HLMP-CB1AXY0DD; Broadcom Inc., San Jose, CA,USA), are inserted at five different angles: $90^{\circ}, 80^{\circ}, 70^{\circ}, 60^{\circ}$, and $45^{\circ}$, relative to the tissue surface (Fig. 1). Red LEDs are arranged in the same plane as the photodiodes, green and blue LEDs are arranged on either side of the red LED offset by $5 \mathrm{~mm}$ perpendicular to the semi-circle plane but still at the same angle as those of the red LEDs. Because the offset is small ( $5 \mathrm{~mm}$ on either side), approximately the same portion of each sample is illuminated by all three LEDs. The tissue stage fits a standard microscope slide to center the tissue below the angular photodiode array. The angular photodiode array height relative to the tissue stage is adjustable, so that the surface of each tissue sample is analyzed despite variations in sample thickness.

\section{B. Circuit Design}

The LEDs were powered by the $5 \mathrm{~V}$ output of an Arduino Mega 2560 microcontroller (SainSmart, Filaments, AB, CA) and driven using an LED driver (6969; Maxim Integrated, San Jose, CA, USA). The outputs from eight PIN photodiodes in photovoltaic mode were collected individually by the same Arduino Mega 2560. The differential output of each photodiode was amplified through a gain stage using quad op-amps (TLC27L4CN; Texas Instruments, Dallas, TX, USA) to amplify the photodiode output and the gain were optimized for each angle of detection so that the broadest range of signal would be detected without saturating the device under normal conditions. Due to the large gain employed to detect our low target signal a high-pass filter was employed to cutoff unwanted signal noise that was substantially amplified as well.

\section{Mie Scattering Simulations for Tissue Components}

Mie scattering simulations were performed using the MiePlot v.4.6 software (Philip Laven; available at www.philiplaven.com/mieplot.htm). Four structural elements were identified that could reasonably alter scatter from heathy to tumor colon tissues: colon cells, colonic crypts, goblet cells, and capillary structure. These structures were simulated for their Mie scattering intensities against the scattering angle and compared to the results of tissue samples, to identify the tissue component(s) dominating or strongly influencing the overall Mie scattering. Each simulation was performed using the inhomogeneous sphere mode and setting the refractive index equation to 3-layer (sinusoidal transitions) in MiePlot so that more structural elements could be better simulated and so that the number of layers could be accounted for. It was reasoned that tubular structures like crypts and capillaries could also be loosely simulated using this method since a ray of light would likely interact with these structures at an angle which nearly simulates an oval. Output Mie spectra was obtained for unpolarized light. Additionally, all simulations assumed a normal distribution with respect to the size of structures with a $10 \%$ standard deviation which is consistent with the range of typical mammalian cells [8]. The selected parameters for each structure can be found in Table 1 . These values were simulated with an angular resolution of $1^{\circ}$ ranging from $110^{\circ}$ to $170^{\circ}$ at 3 different wavelengths of $470 \mathrm{~nm}, 505 \mathrm{~nm}$, and $624 \mathrm{~nm}$. Scattering angles in Mie simulations are defined as $0^{\circ}$ for scattering in the same direction of incident light, $90^{\circ}$ for scattering perpendicular to the incident light, and $180^{\circ}$ for scattering back to the light source, thus $90^{\circ}-180^{\circ}$ as back scatter.

Table 1. Parameters used to model Mie scattering.

\begin{tabular}{llrrr}
\hline $\begin{array}{l}\text { Structural } \\
\text { Components }\end{array}$ & $\begin{array}{l}\text { Sub- } \\
\text { components }\end{array}$ & $\begin{array}{c}\text { Radius } \\
(\mu \mathrm{m})\end{array}$ & $\begin{array}{c}\text { Refractive } \\
\text { index }\end{array}$ & Layer \\
\hline Colon cells & Nucleolus & .5 & 1.42 & 64 \\
& Nucleus & 3.2 & 1.39 & \\
& Cytoplasm & 4.25 & 1.37 & \\
\hline Crypts & Crypt & 25 & 1.333 & 16 \\
& ECM & 35 & 1.375 & \\
\hline Goblet cells & Granules & 5 & 1.35 & 32 \\
& Cytoplasm & 5.5 & 1.37 & \\
\hline Capillaries & Lumen & 5.6 & 1.333 & 8 \\
& ECM & 7 & 1.38 & \\
\hline
\end{tabular}

Data obtained from [9] (colon cells), [10] (crypts and goblet cells) and [11] (capillaries).

\section{Tissue Sample Preparation}

Tumor was induced in a rat model in accordance with an Institutional Animal Care and Use Committee (IACUC) approved protocol (protocol number 17-275). Eight RNU immunodeficient rats (RNU316; Charles River, Wilmington, MA, USA) were purchased. RKO colorectal cancer cells (CRL-2577; ATCC, Manassas, VR, USA) were cultured in EMEM in a cell culture flask and grown to confluence. On the day of injection $10^{6}-10^{7} \mathrm{RKO}$ human cells were suspended in $200 \mu \mathrm{L}$ Eagle's Minimal Essential Medium and maintained on ice prior to injection. Cells are injected subcutaneously into the right flank of RNU immunodeficient rats using a 22-gauge (0.72 mm OD and $0.41 \mathrm{~mm} \mathrm{ID)} \mathrm{or} \mathrm{smaller} \mathrm{needle.} \mathrm{Rats} \mathrm{were}$ 
monitored 1-2 times daily for the next 48 hours until they returned to normal health and activity. Heterotopic colorectal tumors were grown to approximately $2 \mathrm{~cm}$ diameter. Within 6 weeks rats were euthanized and tumors and healthy rat colons were harvested for analysis.

\section{E. Data Collection}

Data was collected in a dark room to reduce the effect of external lighting that might have on measurements. For each sample the device cycled through each of the 15 LEDs illuminating the sample for just over 3 seconds during which time intensity readings were collected every $250 \mathrm{~ms}$ from each of the eight photodiodes and averaged over the three-second window to reduce any signal variation that was a result of noise before moving onto the next LED.

\section{RESULTS AND DISCUSSION}

\section{A. Mie Scattering Simulations}

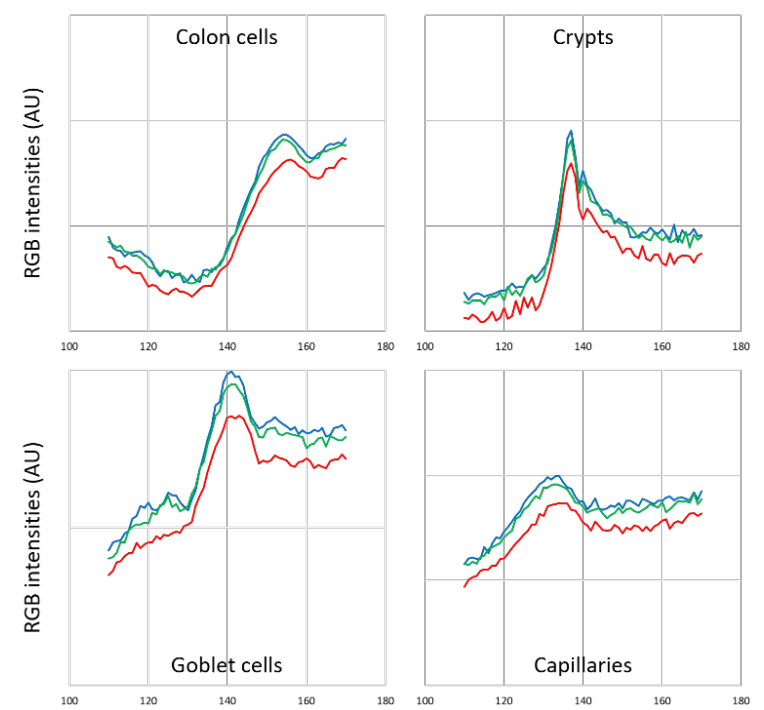

Fig. 2. Simulated Mie scattering profiles ("Mie spectra") for common structures: colon cells, crypts, goblet cells, and capillaries. Red, green and blue lines represent red, green and blue intensities, respectively.

Fig. 2 shows the simulations for the four structural elements that likely would have the biggest impact on Mie scattering in colon tumor: colon cells, crypts, goblet cells, and capillaries. Simulations were conducted for $110^{\circ}-170^{\circ}$ back scattering angles, since detection of forward scattering is not practical with the tissue samples sitting on a flat surface. Colon cells show a peak at slightly higher than $150^{\circ}$ as well as increasing intensities towards lower back scattering angles. Crypts show a sharp peak at slightly lower than $140^{\circ}$. Goblet cells show a sharp peak at $140^{\circ}$, which is similar to those of crypts. Capillaries show a broad peak around $130^{\circ}$. Green and blue intensities are very similar to each other, while red intensities are smaller than them, although the overall shapes are identical for all three colors. Therefore, we expect that our device will likely exhibit a similar pattern.

\section{B. Results with Rat Models}

Fig. 3 shows the average light scattering intensities from the rat specimens. With the blue LED irradiation at $90^{\circ}$, i.e. perpendicular to the surface, no differences are found between tumor and healthy tissue samples. Two peaks can be found, one at $120^{\circ}$ and the other at $150^{\circ}$. Comparing with the simulation results shown in Fig. 2 , the $150^{\circ}$ peak corresponds to that of colon cells and the $120^{\circ}$ peak to the capillaries (and potentially the increasing trend with decreasing scattering angle with colon cells).

With decreasing angle of irradiation $\left(80^{\circ}\right.$ to $\left.45^{\circ}\right)$, more pronounced differences can be found at $150^{\circ}$ between tumor and healthy tissues, while the Mie spectra were not significantly shifted to the left or right. As the intensities at this larger scattering angles $\left(\sim 150^{\circ}\right)$ are always lower with tumor tissues, this can be attributed to the loss of colon cells in tumor tissues. On the other hand, the intensities at smaller angles $\left(\sim 120^{\circ}\right)$ are higher with tumor tissues, which can potentially be attributed to the increase in capillaries with tumor tissues. This angle-dependent amplification rises from many different factors, most likely the Kirchhoff light scattering approximation from rough surfaces [12]: a greater number of secondary scatter events (not affected by incident angle) are observed when a sample is irradiated at high angles of incidence (in our case, low irradiating angles, e.g. $60^{\circ}$ and $45^{\circ}$ ), while light scatter at low angles of incidence (in our case, $80^{\circ}$ and $90^{\circ}$ ) results in more primary scatter events. This effect is likely further complicated because tissue samples exhibit strong scattering from multiple components over the depth of layers.

With green LED irradiation, similar trends can be observed: 1) two peaks at $120^{\circ}$ and $150^{\circ}$ scattering angles, 2) no differences between healthy and tumor tissues with $90^{\circ}$ and $80^{\circ}$ irradiation, and 3) significant differences at the $150^{\circ}$ scattering angle between healthy and tumor tissues. Red LED irradiations are inherently different from the other colors, as blood is essentially transparent to this color, i.e., hemoglobin in red blood cells does not absorb most of red color. As the trend with the red LED irradiations is almost identical to the others, it can indirectly be concluded that the results shown in Fig. 3 are mostly attributed to the Mie scattering of tissue components but not to the absorption of hemoglobin in the tissue samples. However, it should be noted that the results with red LED irradiations show the least significant differences. This can be attributed to 1) the lower scattering intensities in red color (in comparison to blue and green color) as simulated in Fig. 2, and 2) the possible contribution of hemoglobin absorption with blue and green LED irradiations.

\section{CONCLUSION}

Based off our findings we are confident that changes in optical Mie scattering between tumor and healthy colon samples can be detected using a simple photodiode array consisting of just several PIN photodiodes and one or more LEDs. The angular array we designed gives a basis for experimentally identifying angles and colors (wavelengths) of LEDs that are particularly useful for identifying tumor samples from healthy tissue samples despite the fact that tissue specimen exhibits complex scattering behavior. 

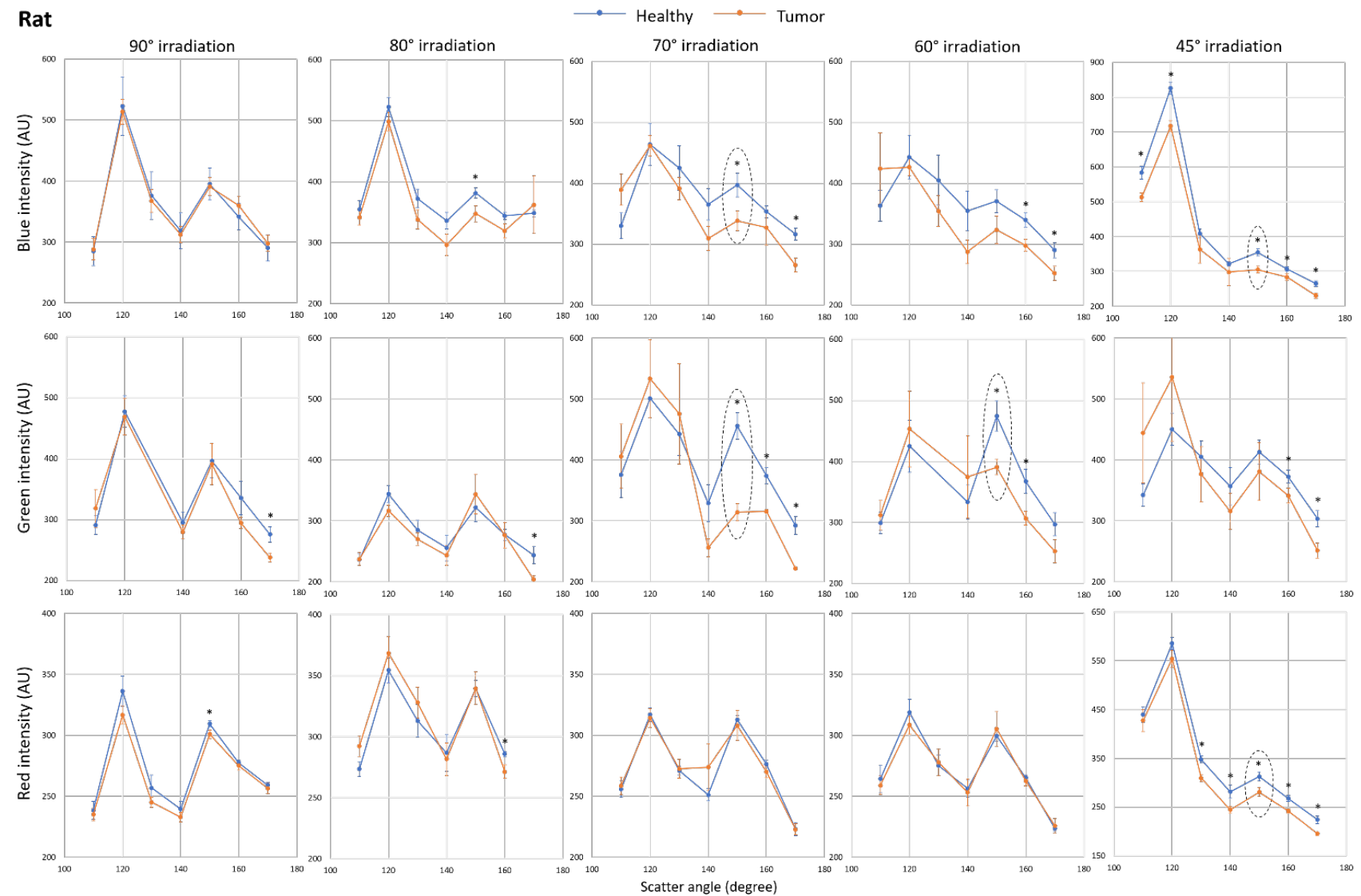

Fig. 3. Mie scattering intensities collected from the angular photodiode array device for healthy and tumor rat tissue samples. Samples were irradiated with blue (top row), green (middle row), or red (bottom row) LEDs positioned at $90^{\circ}$ (perpendicular from the tissue surface), $80^{\circ}, 70^{\circ}, 60^{\circ}$ and $45^{\circ}$. Only one LED irradiated the tissue sample per experiment. Averages from 4 different healthy rat tissue samples and from 6 different tumor rat tissue samples. Error bars represent standard errors. ${ }^{*}$ indicate statistical difference with $p<0.05$ between healthy and tumor tissue samples.

Rat model was employed and tested by growing human RKO tumor cells in immunocompromised RNU rats and the colon tissue samples were harvested. Comparing the Mie scatter simulations to the experimentally collected scatter profile data from the rat model seem to suggest that the scatter profile observed is a product of a number of tissue macro- and microstructural elements where perhaps the two greatest differences in our rat model originating from the loss of colon cells in tumor and the increase in capillaries.

Stronger and collimated LEDs and modulation of the LEDs with lock-in detection can improve the signal to noise ratio and avoid the requirement of a dark room. While the method is primarily designed for in vitro analyses, LED and photodiode pair at optimized angle may be incorporated at the end of an endoscope for in vivo diagnostics.

\section{ACKNOWLEDGMENT}

This work was supported by Tech Launch Arizona, grant number UA16-179, as well as the Biomedical Imaging and Spectroscopy Training Grant from the U.S. National Institutes of Health, grant number T32EB000809.

\section{REFERENCES}

[1] Sweeney R E, Budiman E, Yoon J-Y (2017), "Mie scatter spectra-based device for instant, contact-free, and specific diagnosis of bacterial skin infection," Sci. Rep., vol. 7, p. 4801, doi: 10.1038/s41598-017-05061-1

[2] Sweeney R E, Yoon J-Y (2017), "Angular photodiode array-based device to detect bacterial pathogens in a wound model,” IEEE Sens. J., vol. 17, no. 21, pp. 6911-6917, doi: 10.1109/JSEN.2017.2752155
[3] You D J, Park T S, Yoon J-Y (2013), "Cell-phone-based measurement of TSH using Mie scatter optimized lateral flow assays," Biosens Bioelectron., vol. 40, no. 1, pp. 180-185, doi: 10.1016/j.bios.2012.07.014

[4] Park T S, Li W, McCracken K E, Yoon J-Y (2013), "Smartphone quantifies Salmonella from paper microfluidics," Lab Chip, vol. 13, no. 24, pp. 4832-4840, doi: $10.1039 / \mathrm{c} 31 \mathrm{c} 50976 \mathrm{a}$

[5] Cho S, Park T S, Nahapetian T G, Yoon J-Y (2015), "Smartphone-based, sensitive $\mu \mathrm{PAD}$ detection of urinary tract infection and gonorrhea," Biosens. Bioelectron., vol. 74, pp. 601-611, doi: 10.1016/j.bios.2015.07.014

[6] Tsai M-C, Tsai T-L, Shieh D-B, Chiu H-T, Lee C-Y (2009), "Detecting HER2 on cancer cells by $\mathrm{TiO}_{2}$ spheres Mie scattering," Anal. Chem., vol. 81, no. 18, pp. 75907596, doi: 10.1021/ac900916s

[7] Baselt T, Rudek F, Richter C, Nelsen B, Lasagni A F, Hartmann P (2018), "Detection of structural changes based on Mie-scattering analyses of mouse fibroblast L929 cells before and after necrosis," Proc. SPIE, vol. 10685, p. 106854D, doi: $10.1117 / 12.2307485$

[8] Kulasinghe A, Tran T H P, Blick T, O'Byrne K, Thompson E W, Warkiani M E, Nelson C, Kenny L, Punyadeera C (2017), "Enrichment of circulating head and neck tumour cells using spiral microfluidic technology," Sci. Rep., vol. 7, p. 42517, doi: $10.1038 /$ srep42517

[9] Sung Y, Choi W, Fang-Yen C, Badizadegan K, Dasari R R, Feld M S (2009), "Optical diffraction tomography for high resolution live cell imaging," Opt. Express, vol. 17 , no. 1 , pp. $266-277$, doi: 10.1364/OE.17.000266

[10] Halm D R, Halm S T (1999), "Secretagogue response of goblet cells and columnar cells in human colonic crypts," Am. J. Physiol. Cell Physiol., vol. 277, no. 3, pp C501-C522, doi: 10.1152/ajpcell.1999.277.3.C501

[11] Forster J, Harriss-Phillips W, Douglass M, Bezak E (2017), "A review of the development of tumor vasculature and its effects on the tumor microenvironment," Hypoxia, vol. 5, pp. 21-32, doi: 10.2147/hp.s133231

[12] Bruce N C, Dainty JC (1991), "Multiple scattering from random rough surfaces using the Kirchhoff approximation," J. Mod. Opt., vol. 38, no. 3, pp. 579-590, doi: $10.1080 / 09500349114552811$. 